\title{
Anaemia in inflammatory bowel disease
}

\author{
Martyn Dibb, Sreedhar Subramanian
}

Department of Gastroenterology, Royal Liverpool University Hospital, Liverpool, UK

\section{Correspondence to}

Dr Sreedhar Subramanian, Department of Gastroenterology, Royal Liverpool University Hospital, Prescot Street, Liverpool, L7 9XP, UK; Sreedhar.Subramanian@rlbuht. nhs.uk

Received 8 September 2013 Accepted 2 December 2013 Published Online First 3 January 2014
To cite: Dibb M, Subramanian S. Frontline Gastroenterology 2014;5:190-196.

\section{ABSTRACT}

Anaemia is a common manifestation in inflammatory bowel disease (IBD) and impairs quality of life. Anaemia in IBD is typically caused by iron deficiency or anaemia of chronic disease. Treatment of iron deficiency with oral iron may lead to gastrointestinal intolerance though this may be related to the dose of iron replacement. New intravenous formulations have emerged which allow safe, rapid and effective correction of iron deficiency in IBD. In this article, we provide a review on the topic and cover recent progress in the field for the practicing gastroenterologist.

\section{INTRODUCTION}

Anaemia is a common manifestation in patients with inflammatory bowel disease (IBD) with reported prevalence ranging from $29 \%$ to $81 \%$ (weighted mean 59\%) in inpatients and $6 \%$ to $73 \%$ (weighted mean $16 \%$ ) in outpatients. ${ }^{1-5}$ It is associated with reduced quality of life scores and an increased mortality rate. ${ }^{6} 7$ The presence of anaemia is defined by WHO as a decline in blood haemoglobin to a concentration $<12 \mathrm{~g} / \mathrm{dL} \quad(120 \mathrm{~g} / \mathrm{L})$ in women and $<13 \mathrm{~g} / \mathrm{dL}(130 \mathrm{~g} / \mathrm{L})$ in men. Anaemia in IBD is multifactorial and a list of causes is shown in box 1. Most cases result from a combination of iron deficiency and anaemia of chronic disease (ACD), although less commonly vitamin deficiency, drug induced causes and haemolysis are seen.

\section{CAUSES OF ANAEMIA IN IBD}

\section{Iron deficiency anaemia in IBD}

Iron deficiency anaemia (IDA) in IBD is predominantly due to intestinal blood loss but malabsorption and reduced intake may also play a role. ${ }^{8}{ }^{9}$ An understanding of basic iron physiology is essential to recognise and treat IDA that accompanies IBD.

\author{
Box 1 Causes of anaemia in inflam- \\ matory bowel disease \\ - Iron deficiency \\ - Cobalamin deficiency \\ - Folate deficiency \\ - Anaemia of chronic disease \\ - Drugs \\ - 5-ASA \\ - 6MPIAZA \\ - Sulfasalazine \\ - Haemolysis \\ - Myelodysplasia \\ - Aplastic anaemia \\ - Glucose 6-phosphate dehydrogenase \\ deficiency
}

An individual's iron requirement is based on the balance between intake and loss. A typical adult has approximately $3 \mathrm{~g}$ of storage iron with two-thirds incorporated into haemoglobin and the rest stored as ferritin or haemosiderin. About $20-25 \mathrm{mg}$ of iron is required daily for haem synthesis. ${ }^{10}$ Most of this is met by recycling of iron from senescent erythrocytes with a further 1-2 mg supplied by dietary intake. ${ }^{11}$ The total iron loss averages $1-2 \mathrm{mg} / \mathrm{d}$, the majority lost through the faeces and the remainder via skin, urine or menstrual loss. ${ }^{10}$

The average adult iron intake is $10-15 \mathrm{mg}$ per day of which approximately $10 \%-20 \%$ is absorbed. Dietary iron comes in two forms: haem iron (found in red meat) and non-haem iron (found in vegetable products). Haem iron is better absorbed and accounts for roughly $40 \%$ of absorbed iron. Non-haem iron occurs mainly in its trivalent form $(\mathrm{Fe} 3+)$, which then undergoes enzymatic reduction from ferric iron $(\mathrm{Fe} 3+)$ to the more readily absorbed ferrous iron $(\mathrm{Fe} 2+)$ by brush border ferrireductase in 
the presence of an acidic gastric $\mathrm{pH} .{ }^{10}$ Divalent metal transporter 1 on duodenal epithelium transfers iron across the apical membrane where it is either transferred across the basolateral membrane to reach plasma bound to transferrin or stored as ferritin and eventually excreted as the enterocyte is sloughed. ${ }^{12}$ The absorption of intestinal iron is affected by several factors such as gastric $\mathrm{pH}$, presence of food and various drugs. Body iron stores also regulate iron absorption tightly; in deficient states, almost all of the ingested iron is absorbed whereas in iron-replete conditions, very little is absorbed. This tight regulation is mediated via the expression of hepcidin, a key peptide in iron homeostasis. Hepcidin is a negative regulator of iron absorption by inhibiting iron transport across the gut mucosa while also inhibiting transport of iron out of macrophages. Thus, in states of high hepcidin levels (including inflammatory states), serum iron levels drop because iron is trapped inside macrophages in turn leading to ACD.

\section{Vitamin $B_{12}$ and folate deficiency in IBD}

Vitamin $B_{12}$ and folate are water-soluble vitamins that have roles in DNA damage, repair and metabolism. Vitamin $\mathrm{B}_{12}$ and folate deficiency are common in patients with IBD and may manifest as megaloblastic anaemia. $^{13}$

The prevalence of vitamin $\mathrm{B}_{12}$ deficiency has been reported as $11 \%-24 \%$ and $7.5 \%$ in patients with Crohn's disease and ulcerative colitis, respectively. ${ }^{13-16}$ Vitamin $\mathrm{B}_{12}$ is absorbed by a highly specific receptor complex in the ileum. ${ }^{17}$ Patients with ileal involvement or resection are therefore at particular risk for vitamin $\mathrm{B}_{12}$ deficiency. Vitamin $\mathrm{B}_{12}$ can also be depleted as a consequence of bacterial overgrowth. Low serum vitamin $\mathrm{B}_{12}$ levels (less than $150 \mathrm{pmol} / \mathrm{L}$ ) are used in routine practise to diagnose deficiency although false positive and negatives have been reported with this assay. ${ }^{14} \quad 18 \quad 19$ Vitamin $B_{12}$ has traditionally been replaced intramuscularly although oral replacement with cyanocobalamin $(1000-2000 \mu \mathrm{g})$ appears to be effective in the general population. ${ }^{20}$

Serum folate deficiency has been reported with a prevalence of between $20 \%$ and $28.8 \%$ in patients with predominantly active Crohn's disease. ${ }^{9}{ }^{13} 21$ Red blood cell (RBC) folate may be a more accurate test as it reflects average $\mathrm{RBC}$ folate status over 3 months. There is limited evidence regarding the prevalence of RBC folate deficiency although this appears significantly lower $(0 \%-7 \%)$ than serum folate alone. ${ }^{18} 22$ Measuring serum homocysteine levels have been reported to be useful in the diagnosis of folate deficiency as this gives an indication of the complete metabolic pathway. ${ }^{19}$ However, vitamin $\mathrm{B}_{12}$ deficiency can also cause raised homocysteine levels. Several mechanisms result in folate deficiency including inadequate dietary intake, malabsorption and medication interactions. Inadequate dietary intake is less common in the developed world where folate fortification is used in processed breakfast cereals. Active ileal disease or previous small bowel resections have a demonstrated association with folate deficiency. ${ }^{13}{ }^{15}$ Methotrexate and sulfasalazine can lead to folate deficiency through their action as dihydrofolate reductase inhibitors and by reducing cellular uptake of folate. Screening for folate deficiency is not currently recommended although patients on methotrexate are routinely supplemented with folic acid $5 \mathrm{mg}$ once weekly. ${ }^{23}$

\section{Anaemia of chronic disease}

ACD is thought to result from suppression of erythropoiesis and dysfunctional iron transport which is mediated by inflammatory cytokines. ${ }^{24}$ The degree of anaemia also generally correlates with disease activity. ${ }^{25}$ Erythropoietin (EPO), a glycoprotein hormone secreted by the kidney, regulates erythropoiesis and maintains a steady level of circulating RBCs in the healthy individual. ${ }^{24}$ While serum EPO levels rise in IBD patients with anaemia, this response is inadequate when compared with patients with anaemia without the systemic inflammation resulting from IBD or other chronic inflammatory conditions. ${ }^{26}$

\section{DIAGNOSIS OF IRON DEFICIENCY IN IBD}

The diagnosis of iron deficiency in IBD is often challenging and difficult to distinguish from ACD (summarised in table 1). A microcytic and hypochromic blood picture suggests IDA but a normal mean corpuscular volume does not exclude this. Conversely, microcytosis does not necessarily imply iron deficiency as it can be produced by other causes such as ACD or sideroblastic anaemia. Thus, the diagnosis of IDA requires supporting laboratory studies including iron studies (serum transferrin/total iron binding capacity (TIBC), transferrin saturation and serum iron) and serum ferritin levels.

Serum ferritin levels reflect the iron content stored in the body. In the absence of coexistent inflammation, a reduced serum ferritin is an excellent indicator

Table 1 Laboratory values in IDA, ACD and mixed anaemia

\begin{tabular}{llll}
\hline & IDA & ACD & Mixed anaemia \\
\hline Serum ferritin & $\downarrow$ & $\uparrow$ & $\uparrow$ or $\leftrightarrow$ \\
Serum iron & $\downarrow$ & $\downarrow$ & $\downarrow$ \\
Transferrin & $\downarrow$ & $\downarrow$ or $\leftrightarrow$ & $\downarrow$ \\
Transferrin saturation & $\downarrow$ & $\downarrow$ & $\downarrow$ \\
TIBC & $\uparrow$ & $\downarrow$ & $\uparrow$ or $\leftrightarrow$ \\
STfR & $\uparrow$ & $\downarrow$ or $\leftrightarrow$ & $\uparrow$ \\
STfR-ferritin index & $\uparrow$ & $\downarrow$ & $\uparrow$ \\
CRP or ESR & $\downarrow$ & $\uparrow$ & $\uparrow$ \\
Hepcidin & $\downarrow$ & $\uparrow$ & $\downarrow$ or $\uparrow$ \\
\hline
\end{tabular}

$\downarrow$, decreased; $\uparrow$, increased; $\leftrightarrow$, unchanged; ACD, anaemia of chronic disease; CRP, C reactive protein; ESR, erythrocyte sedimentation rate; IDA, iron deficiency anaemia; sTfR, soluble transferrin receptor; $T I B C$, total iron binding capacity. 
of iron deficiency (normal range 15-200 $\mu \mathrm{g} / \mathrm{L}$ ). A serum ferritin level $<15 \mu \mathrm{g} / \mathrm{L}$ is regarded as an indication of absolute iron deficiency. ${ }^{8}$ However, being an acute phase reactant, ferritin levels go up with inflammation. The presence of raised inflammatory marker levels of up to $100 \mu \mathrm{g} / \mathrm{L}$ indicates iron deficiency. Serum iron is an unreliable marker of iron deficiency as it is influenced by a variety of factors including diurnal variation, inflammatory processes (decreased), malignancy (decreased) and menstrual blood loss (decreased) to name a few.

Serum transferrin (iron transport protein) is not as reliable as serum ferritin for diagnosis of IDA. Serum transferrin level is inversely related to iron stores. Transferrin level is influenced by factors other than iron stores such as inflammation, infection, malignancy, liver disease, nephrotic syndrome, pregnancy and malnutrition. TIBC indicates the maximum amount of iron needed to saturate transferrin. The correlation between TIBC and transferrin is generally considered good though not interchangeable. Although TIBC is cheaper, it is preferable to measure transferrin as there are universally accepted reference ranges for diagnosis of IDA.

Transferrin saturation is a measure of the iron content of circulating transferrin. A transferrin saturation level below 16\% implies a suboptimal supply of iron for erythropoiesis. ${ }^{8}$ Reduced transferrin saturation is highly sensitive $(90 \%)$ for detecting iron deficiency but specificity $(40 \%-50 \%)$ is relatively low. ${ }^{27}$

Soluble transferrin receptors (sTfRs) are transferrin receptors that are shed from the cell membrane and move into the plasma. In IDA, transferrin receptors are upregulated on RBCs, and consequently serum sTfRs levels are elevated. Unlike ferritin and transferrin, chronic inflammation and hepatic damage have no effect on sTfRs which should make it a reliable marker for diagnosing iron deficiency in chronic inflammatory conditions such as IBD. ${ }^{27} 28$ However, sTfRs have been reported to be less accurate than serum ferritin in chronic renal failure. ${ }^{29}$ In a further study of 176 patients with roughly equal number of patients with ACD and IDA, both the sensitivity and specificity of sTfR in IDA were found to be $100 \%$, whereas in ACD, these were $66.6 \%$ and $100 \%$, respectively, suggesting that sTfR is useful in distinguishing IDA from ACD. ${ }^{30}$ In IBD, Oustamanolakis and Koutroubakis reported a sensitivity and specificity of $81 \%$ and $80 \%$ respectively at a cut-off of $>1.8 \mathrm{mg} / \mathrm{dL}$ for diagnosis of iron deficiency. ${ }^{31}$

The sTfR to log ferritin ratio has also been reported to differentiate more accurately between IDA and ACD or to assess the iron status in patients with mixed type anaemia. ${ }^{32} 33$ The sTfR to log ferritin ratio is a useful alternative to invasive bone marrow examination, but sTfR measurement is expensive and not routinely available. The sTfR-ferritin index has a sensitivity of $91 \%$ and specificity of $92 \%$ in diagnosing iron deficiency in IBD. ${ }^{31}$

A variety of other indices such as zinc protoporphyrin, reticulocyte haemoglobin content, red cell distribution width, red cell size factor and novel factors such as hepcidin and prohepcidin add value to distinguishing IDA from ACD. However, this is beyond the scope of the article; these are reviewed by Oustamanolakis and colleagues. ${ }^{34}$

\section{INVESTIGATION OF ANAEMIA}

Anaemia workup should be initiated if the haemoglobin value is low. The minimum workup should include serum ferritin, transferrin saturation and $\mathrm{C}$ reactive protein concentration. If these fail to yield a cause, further tests including serum transferrin, vitamin $\mathrm{B}_{12}$, folic acid, haptoglobin, lactate dehydrogenase, and creatine, a reticulocyte, and a differential white blood cell count should be performed. Advice from a haematologist is appropriate if the cause of anaemia remains unclear after more extensive workup.

\section{MANAGEMENT OF ANAEMIA}

Iron supplementation should be initiated when IDA is present. However, treatment of the non-anaemic iron deficient patient depends on the clinical scenario and risks and benefits of treatment need to be discussed with the patient. The goals of treatment are to achieve normal levels of haemoglobin, serum ferritin and transferrin saturation above the lower threshold of normal, avoid the use of blood transfusion, relieve symptoms related to anaemia, and improve the quality of life.

Although iron is commonly prescribed, the amount of elemental iron needed to achieve clinical efficacy and the optimal method of supplementation are under debate. As intravenous iron supplementation has become safer, calls for increased utilisation have appeared. ${ }^{1} 28$ However, there are significant cost implications to using intravenous iron. Moreover, oral iron has been shown to be effective in treating IDA associated with IBD. De Silva and colleagues conducted a prospective open-label study with 33 IBD patients and 14 non-IBD patients with iron-deficiency anaemia. ${ }^{35}$ Patients were treated with $200 \mathrm{mg}$ ferrous sulfate three times a day (each tablet contains $60 \mathrm{mg}$ of elemental iron). At the end of 4 weeks, haemoglobin levels had a statistically significant increase in all groups, an increase from 10 to 11.7 in the Crohn's disease group $(n=19), 12.3$ to 12.7 in the ulcerative colitis group $(n=14)$ and 10.2 to 11.9 in the non-IBD group $(n=14)$. In IBD patients with active disease, the mean increase in haemoglobin was from 10 to 13.2 $(n=7)$. The incidence of gastrointestinal side effects was about $25 \%$ in each group, similar to what has been reported in the general population. ${ }^{36} \mathrm{~A}$ randomised open-label study compared treatment of 41 
iron-deficient IBD patients with $100 \mathrm{mg}$ ferrous sulfate twice a day versus a ferric form of iron, ironpolymaltose complex. After 2 weeks, there was no increase in clinical disease activity in Crohn's disease or ulcerative colitis patients though a change in antioxidant status was noted. ${ }^{37}$ Several other concerns have been raised in relation to oral iron therapy in IBD including poor absorption especially in the presence of active disease, its potential to exacerbate IBD, generation of reactive oxygen species, prolonged duration of therapy to achieve optimal effect and the ability of iron to worsen inflammation and induce carcinogenesis in animal models of colitis. However, some of these concerns have not been adequately substantiated.

Another factor associated with intolerance of oral iron may be related to the dose of elemental iron administered. Most studies investigating the efficacy of oral iron have used a typical dose of $150-200 \mathrm{mg} / \mathrm{d}$ of elemental iron, a 10-20-fold in excess of daily intake. Due to the $20 \%$ incidence level of intolerance at conventional doses of elemental iron, recent studies have investigated the efficacy and side effects associated with low-dose oral iron supplementation. ${ }^{36}$ A study in the elderly (age >80) randomised 90 patients with IDA to 15,50 or $150 \mathrm{mg}$ of daily oral elemental iron over 2 months. All three dosage groups experienced a similar, statistically significant increase in haemoglobin after 2 months. ${ }^{36}$ These studies have not been done in IBD. Overall, oral iron supplementation is efficacious in patients with IBD, with a similar incidence of side effects in comparison with patients without IBD and no convincing evidence of clinical deterioration in symptoms or disease activity.

\section{Intravenous iron administration}

Intravenous iron therapy is recommended for patients with a lack of efficacy or intolerance to oral iron; for patients with severe anaemia (haemoglobin level $<10 \mathrm{~g} / \mathrm{dL}$ ); for patients with severe disease; or for patients treated with EPO. Some of the earlier intravenous iron preparations were limited by toxicity but have been superseded by newer preparations. The characteristics of the commonly available iron preparations in the UK are summarised in table 2.

Intravenous iron is effective and well tolerated in IBD patients. ${ }^{38-40}$ A recent systematic review and meta-analysis found a total of 10 clinical studies evaluating iron treatment in IBD including three randomised clinical trials. ${ }^{41}$ The authors concluded that iron is at least as well tolerated and more effective than oral iron in improving ferritin, whereas the differences in haemoglobin values were small and of uncertain clinical relevance. A limited analysis on quality of life data found no relevant differences. Early clinical trials in IBD were reported using iron sucrose but it requires $5-10$ infusions as only a maximum of $200 \mathrm{mg}$ is administered in a single infusion. ${ }^{36} 4042$

In a randomised study of 137 patients, Kulnigg et al ${ }^{39}$ reported that iron carboxymaltose, which usually requires one to two infusions, was also effective and well tolerated in IBD patients. This was followed by a larger, randomised, clinical trial (FERGIcor study) comparing iron sucrose with ferric carboxymaltose in 485 IBD patients. ${ }^{43}$ Iron carboxymaltose was more effective than iron sucrose both in reaching target haemoglobin levels (65.8 vs $53.6 \%$ ) and in correcting anaemia $(83.8 \%$ vs $75.9 \%)$, and required fewer infusions $(2.1$ vs 5.8 infusions, $\mathrm{p}<0.001)$. A costeffectiveness analysis showed that iron carboxymaltose was cheaper than iron sucrose ( $\$ 653$ vs $\$ 891$ ) due to the higher number of infusions with iron sucrose.

Recurrence of anaemia is a significant issue in patients treated with iron supplementation. In a meta-analysis of 88 patients, anaemia recurred in 50\% of patients who had a normal haemoglobin after treatment at a median of 10 months. ${ }^{44}$ In order to assess the efficacy of ferric carboxymaltose (FCM) in preventing recurrence of anaemia, patients from the FERGIcor study were subsequently entered in a follow-up study where patients were given either iron carboxymaltose or placebo when the ferritin levels dropped to less than $100 \mu \mathrm{g} / \mathrm{L}{ }^{45}$ Anaemia recurred in $26.7 \%$ of subjects given FCM and in $39.4 \%$ given placebo at 8 months. Changes in quality of life and disease activity were comparable between groups. In addition, iron carboxymaltose also reduced platelet counts and platelet activation in patients with IBD-associated secondary thrombocytosis in a small single blind placebo controlled trial. ${ }^{46}$ However, this effect is also observed with other intravenous iron preparations. ${ }^{37}$ Other iron preparations such as low molecular weight iron dextran and iron isomaltoside have shown favourable results though large trial data are lacking. ${ }^{4748}$ Bhandari published a cost model of the various iron preparations for correction of IDA and

Table 2 Characteristics of available intravenous iron formulations

\begin{tabular}{lllll}
\hline Molecular composition & Iron dextran & Iron sucrose & Iron carboxymaltose & Iron isomaloside \\
\hline Single dose iron correction & Yes & No & Not if dose exceeds $1 \mathrm{~g}$ & Yes \\
Maximum dose per infusion & $20 \mathrm{mg} / \mathrm{kg}$ & $200 \mathrm{mg}$ & Up to 20 mg/kg (maximum dose $1 \mathrm{~g})$ & $20 \mathrm{mg} / \mathrm{kg}$ \\
Need for test dose & Yes & Yes & No & No \\
Infusion time & $4-6 \mathrm{~h}$ & Up to 15 min & $15 \mathrm{~min}$ & $1 \mathrm{~h}$ \\
\hline
\end{tabular}


showed iron isomaltoside and low molecular weight iron dextran to be less expensive than iron sucrose and iron carboxymaltose but low molecular weight iron dextran has to be infused over several hours and reduces throughput. ${ }^{49}$ Despite its excellent efficacy, intravenous iron may not always be effective and can be improved by using EPO in selected, refractory cases. ${ }^{7}$

\section{Erythropoietin}

A number of erythropoietic agents are available in clinical practise: epoetin $\alpha$, epoetin $\beta$, darbepoietin $\alpha$ and methoxy polyethylene glycol-epoetin $\beta$ (a continuous EPO receptor activator). A number of studies have reported efficacy of EPO in patients with IBD. ${ }^{42}$ 50-52 EPO replacement has been shown to cause a greater increase in haemoglobin levels than iron replacement and combination therapy with EPO and iron is superior to iron therapy alone. ${ }^{52}{ }^{53}$ Darbepoetin and continuous EPO receptor activators have the potential advantages of less frequent administration and greater patient convenience. It should be noted that the cost of all erythropoietic agents is significantly greater than that of intravenous iron replacement and therefore should only be considered in those patients unresponsive to iron replacement or with low EPO levels. EPO treatment should always be an adjunct and not a substitute to appropriate treatment of IBD aimed at suppression of inflammation and mucosal healing. A suggested algorithm for the treatment of IDA is shown in figure 1.

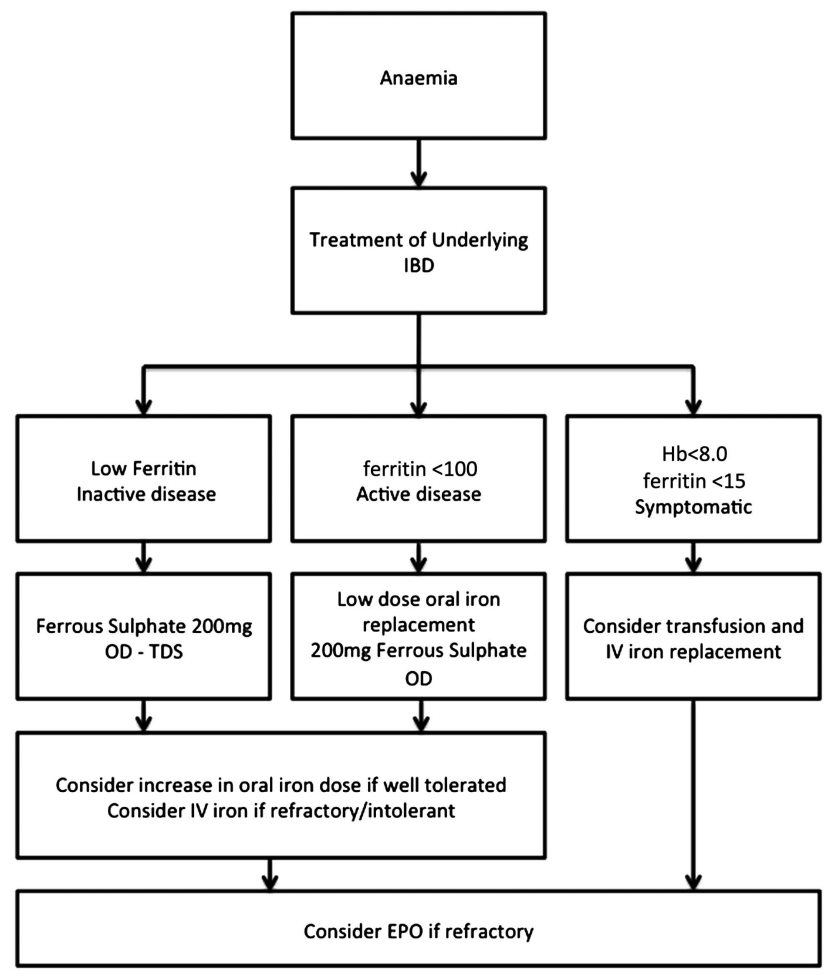

Figure 1 Iron deficiency anaemia treatment algorithm in inflammatory bowel disease (IBD).

\section{CONCLUSIONS}

Anaemia is common in IBD patients. The most common causes are iron deficiency and ACD, although other causes need to be excluded. Assessment and treatment for active disease followed by careful and thorough diagnostic evaluation of the anaemia are essential. This is essential to establish the causation and treatment strategy. High dose oral iron is effective but may be limited by side effects. Intravenous iron is safe and generally well tolerated. Combination with erythropoetic medications should be reserved for refractory cases.

Contributors Both authors performed a literature search, wrote sections of the article and edited the article.

Competing interests None.

Provenance and peer review Not commissioned; externally peer reviewed.

\section{REFERENCES}

1 Gisbert JP, Gomollón F. Common misconceptions in the diagnosis and management of anemia in inflammatory bowel disease. Am J Gastroenterol 2008;103:1299-307.

2 Rejler M, Tholstrup J, Andersson-Gäre B, et al. Low prevalence of anemia in inflammatory bowel disease: A population-based study in Sweden. Scand J Gastroenterol 2012;47:937-42.

3 Oustamanolakis P, Koutroubakis IE, Messaritakis I, et al. Measurement of reticulocyte and red blood cell indices in the evaluation of anemia in inflammatory bowel disease. J Crohns Colitis 2011;5:295-300.

4 Voegtlin M, Vavricka SR, Schoepfer AM, et al. Prevalence of anaemia in inflammatory bowel disease in Switzerland: a cross-sectional study in patients from private practices and university hospitals. J Crohns Colitis 2010;4:642-8.

5 Bager P, Befrits R, Wikman O, et al. The prevalence of anemia and iron deficiency in IBD outpatients in Scandinavia. Scand J Gastroenterol 2011;46:304-9.

6 Wilson A, Reyes E, Ofman J. Prevalence and outcomes of anemia in inflammatory bowel disease: a systematic review of the literature. Am J Med 2004;116(Suppl 7A):44S-9S.

7 Gasche C, Lomer MCE, Cavill I, et al. Iron, anaemia, and inflammatory bowel diseases. Gut 2004;53:1190-7.

8 Gasche C, Berstad A, Befrits R, et al. Guidelines on the diagnosis and management of iron deficiency and anemia in inflammatory bowel diseases\#. Inflamm Bowel Dis 2007;13:1545-53.

9 Hodges P, Gee M, Grace M, et al. Vitamin and iron intake in patients with Crohn's disease. J Am Diet Assoc 1984;84:52-8.

10 Rizvi S, Schoen RE. Supplementation with oral vs. intravenous iron for anemia with IBD or gastrointestinal bleeding: is oral iron getting a bad rap? Am J Gastroenterol 2011;106:1872-9.

11 Heeney MM, Andrews NC. Iron homeostasis and inherited iron overload disorders: an overview. Hematol Oncol Clin North Am 2004;18:1379-403.

12 Fleming RE, Bacon BR. Orchestration of Iron Homeostasis. N Engl J Med 2005;352:1741-4.

13 Yakut M, Üstün Y, Kabaçam G, et al. Serum vitamin B12 and folate status in patients with inflammatory bowel diseases. Eur J Intern Med 2010;21:320-3.

14 Wagner DA, Schatz R, Coston R, et al. A new ${ }^{13} \mathrm{C}$ breath test to detect vitamin B12 deficiency: a prevalent and poorly diagnosed health problem. J Breath Res 2011;5:046001. 
15 Vagianos K, Bernstein CN. Homocysteinemia and B vitamin status among adult patients with inflammatory bowel disease: a one-year prospective follow-up study. Inflamm Bowel Dis 2012;18:718-24.

16 Headstrom PD, Rulyak SJ, Lee SD. Prevalence of and risk factors for vitamin $\mathrm{B}(12)$ deficiency in patients with Crohn's disease. Inflamm Bowel Dis 2008;14:217-23.

17 Kozyraki R, Cases O. Vitamin B12 absorption: mammalian physiology and acquired and inherited disorders. Biochimie 2013;95:1002-7.

18 Hwang C, Ross V, Mahadevan U. Micronutrient deficiencies in inflammatory bowel disease: from A to zinc. Inflamm Bowel Dis 2012;18:1961-81.

19 Klee GG. Cobalamin and folate evaluation: measurement of methylmalonic acid and homocysteine vs vitamin B12 and folate. Clin Chem 2000;46:1277-83.

20 Vidal-Alaball J, Butler C, Cannings-John R, et al. Oral vitamin B12 versus intramuscular vitamin B12 for vitamin B12 deficiency. In: The Cochrane Collaboration, Vidal-Alaball J, eds. Cochrane database of systematic reviews. Chichester, UK: John Wiley \& Sons, Ltd, 2005. http://doi.wiley.com/10.1002/ 14651858.CD004655.pub2 (accessed 6 Aug 2013).

21 Fernandez-Banares F, Abad-Lacruz A, Xiol X, et al. Vitamin status in patients with inflammatory bowel disease. Am J Gastroenterol 1989;84:744-8.

22 Vagianos K, Bector S, McConnell J, et al. Nutrition assessment of patients with inflammatory bowel disease. J Parenter Enter Nutr 2007;31:311-9.

23 Rampton DS. Methotrexate in Crohn's disease. Gut 2001;48:790-1.

24 Tsiolakidou G, Koutroubakis IE. Stimulating erythropoiesis in inflammatory bowel disease associated anemia. World J Gastroenterol 2007;13:4798.

25 Cronin CC, Shanahan F. Anemia in patients with chronic inflammatory bowel disease. Am J Gastroenterol 2001;96:2296-8.

26 Johannsen H, Jelkmann W, Wiedemann G, et al. Erythropoietin/haemoglobin relationship in leukaemia and ulcerative colitis. Eur J Haematol 1989;43:201-6.

27 Wish JB. Assessing iron status: beyond serum ferritin and transferrin saturation. Clin J Am Soc Nephrol 2006;1:S4-8.

28 Stein J, Hartmann F, Dignass AU. Diagnosis and management of iron deficiency anemia in patients with IBD. Nat Rev Gastroenterol Hepatol 2010;7:599-610.

29 Fernández-Rodríguez AM, Guindeo-Casasús MC, Molero-Labarta T, et al. Diagnosis of iron deficiency in chronic renal failure. Am J Kidney Dis Off J Natl Kidney Found 1999;34:508-13.

30 Hanif E, Ayyub M, Anwar M, et al. Evaluation of serum transferrin receptor concentration in diagnosing and differentiating iron deficiency anaemia from anaemia of chronic disorders. JPMA J Pak Med Assoc 2005;55:13-6.

31 Oustamanolakis P, Koutroubakis IE. Soluble transferrin receptor-ferritin index is the most efficient marker for the diagnosis of iron deficiency anemia in patients with IBD. Inflamm Bowel Dis 2011;17:E158-159.

32 Jain S, Narayan S, Chandra J, et al. Evaluation of serum transferrin receptor and sTfR ferritin indices in diagnosing and differentiating iron deficiency anemia from anemia of chronic disease. Indian J Pediatr 2010;77:179-83.

33 Thomas L, Thomas C. Anemia in iron deficiency and disorders of iron metabolism. Dtsch Med Wochenschr 1946 2002;127:1591-4.
34 Oustamanolakis P, Koutroubakis IE, Kouroumalis EA. Diagnosing anemia in inflammatory bowel disease: beyond the established markers. J Crohns Colitis 2011;5:381-91.

35 De Silva AD, Tsironi E, Feakins RM, et al. Efficacy and tolerability of oral iron therapy in inflammatory bowel disease: a prospective, comparative trial. Aliment Pharmacol Ther 2005;22:1097-105.

36 Schröder O, Mickisch O, Seidler U, et al. Intravenous iron sucrose versus oral iron supplementation for the treatment of iron deficiency anemia in patients with inflammatory bowel disease--a randomized, controlled, open-label, multicenter study. Am J Gastroenterol 2005;100:2503-9.

37 Kulnigg-Dabsch S, Evstatiev R, Dejaco C, et al. Effect of iron therapy on platelet counts in patients with inflammatory bowel disease-associated anemia. PLoS ONE 2012;7:e34520.

38 Erichsen K, Ulvik RJ, Nysaeter G, et al. Oral ferrous fumarate or intravenous iron sucrose for patients with inflammatory bowel disease. Scand J Gastroenterol 2005;40:1058-65.

39 Kulnigg S, Stoinov S, Simanenkov V, et al. A novel intravenous iron formulation for treatment of anemia in inflammatory bowel disease: the ferric carboxymaltose (FERINJECT) randomized controlled trial. Am J Gastroenterol 2008;103:1182-92.

40 Lindgren S, Wikman O, Befrits R, et al. Intravenous iron sucrose is superior to oral iron sulphate for correcting anaemia and restoring iron stores in IBD patients: a randomized, controlled, evaluator-blind, multicentre study. Scand J Gastroenterol 2009;44:838-45.

41 Lee TW, Kolber MR, Fedorak RN, et al. Iron replacement therapy in inflammatory bowel disease patients with iron deficiency anemia: a systematic review and meta-analysis. J Crohns Colitis 2012;6:267-75.

42 Koutroubakis IE, Karmiris K, Makreas S, et al. Effectiveness of darbepoetin-alfa in combination with intravenous iron sucrose in patients with inflammatory bowel disease and refractory anaemia: a pilot study. Eur J Gastroenterol Hepatol 2006;18:421-5.

43 Evstatiev R, Marteau P, Iqbal T, et al. FERGIcor, a randomized controlled trial on ferric carboxymaltose for iron deficiency anemia in inflammatory bowel disease. Gastroenterology 2011;141:846-53.e2.

44 Kulnigg S, Teischinger L, Dejaco C, et al. Rapid recurrence of IBD-associated anemia and iron deficiency after intravenous iron sucrose and erythropoietin treatment. Am J Gastroenterol 2009;104:1460-7.

45 Evstatiev R, Alexeeva O, Bokemeyer B, et al. Ferric carboxymaltose prevents recurrence of anemia in patients with inflammatory bowel disease. Clin Gastroenterol Hepatol Off Clin Pract J Am Gastroenterol Assoc 2013;11:269-77.

46 Kulnigg-Dabsch S, Schmid W, Howaldt S, et al. Iron deficiency generates secondary thrombocytosis and platelet activation in IBD: the randomized, controlled thromboVIT trial. Inflamm Bowel Dis 2013;19:1609-16.

47 Koutroubakis IE, Oustamanolakis P, Karakoidas C, et al. Safety and efficacy of total-dose infusion of low molecular weight iron dextran for iron deficiency anemia in patients with inflammatory bowel disease. Dig Dis Sci 2010;55: 2327-31.

48 Nordfjeld K, Andreasen H, Thomsen LL. Pharmacokinetics of iron isomaltoside 1000 in patients with inflammatory bowel disease. Drug Des Devel Ther 2012;6:43-51.

49 Bhandari S. Update of a comparative analysis of cost minimization following the introduction of newly available 
intravenous iron therapies in hospital practice. Ther Clin Risk Manag 2011;7:501-9.

50 Liu S, Ren J, Li J. Response efficacy of erythropoietin combined with enteral nutrition for the treatment of anemia in Crohn's disease. Nutr Clin Pract 2013; 28:523.

51 Horina JH, Petritsch W, Schmid CR, et al. Treatment of anemia in inflammatory bowel disease with recombinant human erythropoietin: results in three patients. Gastroenterology 1993;104:1828-31.

52 Schreiber S, Howaldt S, Schnoor M, et al. Recombinant erythropoietin for the treatment of anemia in inflammatory bowel disease. N Engl J Med 1996;334:619-24.

53 Gasché C, Dejaco C, Waldhoer T, et al. Intravenous iron and erythropoietin for anemia associated with Crohn disease. A randomized, controlled trial. Ann Intern Med 1997;126:782-7. 